Meta

Journal des tradlucteurs

Translators' Journal

\title{
Le registre littéraire en interprétation simultanée
}

\section{Karla Déjean le Féal}

Volume 30, numéro 1, mars 1985

Interprétation de conférence

URI : https://id.erudit.org/iderudit/003448ar

DOI : https://doi.org/10.7202/003448ar

Aller au sommaire du numéro

Éditeur(s)

Les Presses de l'Université de Montréal

ISSN

0026-0452 (imprimé)

1492-1421 (numérique)

Découvrir la revue

Citer cet article

Déjean le Féal, K. (1985). Le registre littéraire en interprétation simultanée.

Meta, 30(1), 55-64. https://doi.org/10.7202/003448ar d'utilisation que vous pouvez consulter en ligne.

https://apropos.erudit.org/fr/usagers/politique-dutilisation/ 


\title{
LE REGISTRE LITTÉRAIRE EN INTERPRÉTATION SIMULTANÉE
}

\author{
Karla DéJean le FÉAL
}

La plupart des communications orales ou préalablement rédigées, présentées dans les réunions internationales, se situent à un niveau de langue moyen. J'entends par là le registre dans lequel un enseignant fait son cours, par exemple, ou encore celui des informations radio-diffusées. Ce niveau qui participe à la fois de la langue soutenue et de la langue commune' est tout d'abord la conséquence de la nature des sujets traités d'ordinaire dans les conférences internationales. Personne n'aurait, en effet, l'idée de briguer les palmes académiques en vertu d'un exposé sur la circulation extra-corporelle en chirurgie infantile, par exemple, ou sur les avantages d'un nouveau transporteur à secousses.

En outre, ce registre moyen correspond aux habitudes d'expression des membres de la couche socio-culturelle dans laquelle se recrute la majorité des conférenciers.

Pour les mêmes raisons, le niveau de langue moyen constitue le registre habituel des interprètes. Leur milieu socio-culturel est analogue à celui de la plupart des intervenants, et leur façon de s'exprimer est modelée par leur travail quotidien qui les oblige à discourir sur des sujets exigeant précisément un registre moyen. C'est celui-là qui leur est le plus familier et dans lequel ils se sentent le plus à l'aise. En traduisant des exposés formulés dans ce registre moyen, ils sont donc à même de s'exprimer avec un maximum de spontanéité et de naturel.

Mais il y a également des discours dont le registre se situe soit en deçà, soit au-delà de ce niveau moyen. L'interprète, tenu de conformer son niveau de langue à celui de l'original, ne peut plus laisser libre cours à ses habitudes d'expression, mais doit emprunter un langage dont il se sert moins souvent dans l'exercice de son métier et que, de ce fait, il ne manie pas avec la même facilité.

Pourtant, il ne peut se soustraire à cette contrainte. S'il veut être fidèle, il doit nécessairement user du même registre que l'orateur. Je dirais même que l'inobservation du registre original conduit à une infidélité de la traduction plus grave et plus lourde de conséquences que celle causée par une erreur matérielle que l'interprète pourrait commettre. En effet, lorsqu'il y a erreur matérielle et que celle-ci est très grossière, les auditeurs, plus experts que l'interprète, la redressent spontanément et peut-être même inconsciemment. Un interprète, par exemple, qui se tromperait d'unité de mesure parce que trop peu informé pour évaluer les ordres de grandeur, et qui dirait à des toxicologues que le résultat moyen du dosage de plomb effectué sur 34 boîtes de sardines à l'huile était de 0,864 grammes par 100 grammes ne risquerait guère de semer la confusion ou de provoquer des malentendus parmi les participants. Ceux-ci remplaceraient aussitôt les grammes par des milligrammes.

Si l'erreur matérielle est plus discrète, elle est susceptible de déclencher une discussion qui permet à l'orateur de dissiper le malentendu.

Dans les deux cas, l'interprète sera identifié comme l'auteur de l'erreur et celle-ci, pour regrettable qu'elle soit, n'aura pas fait beaucoup de dégâts. 
En revanche, quand l'interprète adopte un registre différent de celui de l'original'2, l'auditeur ne conclut pas forcément à une insuffisance de la traduction. L'idée que l'interprétation simultanée consiste en un simple transcodage est bien trop enracinée même chez les habitués des réunions internationales pour que les fautes de registre soient attribuées à l'interprète. L'auditeur sera vraisemblablement davantage enclin à en faire endosser la paternité à l'orateur sans lui fournir, cette fois-ci, l'occasion de se justifier. Pourtant, traduit dans un registre inadéquat, le meilleur discours est transformé en platitudes ou tourné en ridicule. Pis, contrairement à l'erreur matérielle qui, dans l'hypothèse la plus défavorable, ne pourrait mettre en doute qu'un élément du savoir de l'orateur, le registre inapproprié de la traduction risque de jeter le discrédit sur toute la personnalité de l'orateur. Ne dit-on pas que le ridicule tue?

Le respect du registre de l'original est donc un impératif absolu pour l'interprète. On voit aussi que le problème qu'il est susceptible de lui poser concerne essentiellement la réexpression.

Parmi les registres qui se situent en deçà ou au-delà du niveau moyen, le registre littéraire est le plus fréquent dans les réunions internationales. On ne le rencontre pratiquement que dans les discours écrits. Or, l'interprétation est toujours une improvisation et toute improvisation va de pair avec diverses incorrections du langage, qui ne sont guère compatibles avec le style littéraire.

Il en découle un premier problème pour l'interprète : comme il est, par définition, forcé d'improviser, sa version du discours n'a que peu de chances d'atteindre le niveau stylistique de l'original dont l'auteur a peut-être mis des jours à polir la forme. Par conséquent, l'interprète part perdant.

Le deuxième handicap qu'il doit affronter est le maniement du langage littéraire, langue noble qu'il ne parle pas avec une parfaite spontanéité.

Toutefois - et c'est là une constatation qui m'a toujours intriguée - l'interprète est généralement beaucoup plus à l'aise lorsqu'il traduit des discours littéraires en consécutive qu'il ne l'est en simultanée. Comme, dans les deux cas, il doit improviser et que le registre qu'il lui faut employer est le même, la différence doit être due à d'autres causes.

D'après mes observations, il semble, en effet, possible de dégager au moins deux causes : d'une part, la difficulté particulière que soulève la traduction simultanée des expressions imagées qui émaillent souvent les discours littéraires, et, d'autre part, la manifestation particulièrement gênante d'un phénomène propre à l'interprétation simultanée, à savoir l'affaiblissement passager du sens de la langue maternelle chez l'interprète.

Voyons successivement ces deux facteurs.

\section{LES EXPRESSIONS IMAGÉES}

Lorsqu'on exploite des enregistrements de discours littéraires et leur interprétation, on constate que dans bon nombre de cas - surtout si l'interprète n'a pas pu, au préalable, prendre connaissance du texte à interpréter et que celui-ci est lu à un rythme rapide - une grande partie des métaphores utilisées par l'orateur ne se retrouve pas dans la version interprétée.

Il y a donc lieu de s'interroger sur les raisons pour lesquelles l'interprète tend à sacrifier ces expressions imagées. L'explication que je propose est la suivante :

Les métaphores constituent des combinaisons d'éléments linguistiques, que leur auteur crée en fonction des images qu'il entend évoquer, mais qui doivent néanmoins obéir aux contraintes de la langue, notamment à celles de faire comprendre l'idée qu'elles sont censées illustrer et de répondre au génie de la langue et à ses canons de beauté. 
Une des composantes de la métaphore, à savoir l'image, en tant que telle, a donc un caractère universel, indépendant de toute langue, alors que le choix de cette image et la forme verbale sous laquelle elle est évoquée sont intimement liés à la langue.

\section{a) L'effet de style}

Comme les métaphores sont utilisées à des fins stylistiques, c'est l'effet de style réalisable dans une langue par l'évocation de telle image qui détermine le choix de cette image.

Les langues étant différentes les unes des autres, telle image qui se prête à un effet de style dans une langue ne peut être exploitée avec le même bonheur dans une autre. Les proverbes et locutions sont là pour nous le rappeler.

Toutefois, contrairement aux proverbes et aux locutions, les métaphores ne sont pas des phrases figées. L'interprète ne peut donc pas savoir d'avance si, pour obtenir le même effet stylistique dans la langue cible, il est possible ou non de conserver l'image. En effet, en entendant la maxime : Il ne faut pas vendre la peau de l'ours avant de l'avoir tué, il n'a pas besoin de se poser cette question. Il sait s'il y a un proverbe équivalent dans la langue cible, et, dans l'affirmative, il n'a plus qu'à le réciter.

Il n'en va pas de même de l'énoncé suivant ${ }^{3}:$ Je crois que la réponse va de soi. Seul le nombre fait la force. Quelques miettes du pouvoir, ce n'est pas le pouvoir. Un vague saupoudrage des assemblées parlementaires ou des conseils ministériels, ce n'est pas le pouvoir. Il faut, je crois, absolument passer du féminin singulier au féminin pluriel, assurer un véritable décollage de la participation des femmes à la vie publique.

Pour pouvoir décider du maintien des images ou de leur substitution, il faudrait que l'interprète puisse faire un ou plusieurs essais de formulation à blanc, comme lors d'une rédaction. Il n'en a évidemment pas le temps.

Cette incertitude qu'il éprouve en face de la métaphore explique sans doute la disparition, dans la traduction, d'un certain nombre d'entre elles, même lorsque leur transposition ne pose aucun problème. Si l'interprète s'abstient, même en l'absence de difficultés réelles, c'est, à mon avis, parce qu'il ne veut pas se lancer dans l'aventure à l'aveuglette et risquer d'alourdir le discours par des essais de style avortés.

\section{b) La rémanence de l'image}

Lorsque l'image n'est pas transposable, et à supposer même que l'interprète le sente d'emblée, il est peu probable qu'il parvienne à y substituer une autre. En effet, la représentation mentale que la première a fait naître chez lui empêcherait d'en faire immédiatement surgir une autre plus appropriée. Comme la représentation mentale engendrée par une expression imagée persiste plus longtemps dans sa mémoire que les mots prononcés, et que l'image, nous l'avons vu, est choisie en fonction des données de la langue originale, c'est par l'intermédiaire de l'image que l'interprète reste, d'une certaine manière, prisonnier de la langue source.

Quand il a affaire à un énoncé non métaphorique, c'est le sens à l'état de concept qu'il réexprime. La restitution du message peut donc, a priori, être tout à fait indépendante de sa forme originale. La métaphore en revanche se soustrait à ce traitement en raison de la rémanence de l'image.

Cette rémanence est due à l'inédit de l'association que la métaphore établit entre telle image et tel concept. En effet, beaucoup de mots et de locutions toutes faites ne sont pas moins imagés que les métaphores. Le mot résineux, par exemple, ou son équivalent en allemand Nadelhölzer suggèrent, eux aussi, des images de l'objet qu'ils désignent. Pourtant un Français qui entend parler de résineux ne voit pas couler la sève visqueuse mais pense à une catégorie d'arbres. Il en est de même pour l'Allemand dont la repré- 
sentation mentale ne s'arrête pas aux aiguilles, trait saillant de Nadelhölzer. C'est que l'association fréquente entre l'image qu'évoque la désignation de l'objet et cet objet luimême fait perdre peu à peu à l'image son caractère frappant. Plus on est familiarisé avec l'objet ou le concept et sa désignation, moins on se rend compte de la nature imagée de la désignation et de sa signification propre.

Ainsi, lorsqu'on suit une conversation où l'échange des propos se fait, par exemple, dans deux langues qu'on comprend sans difficultés, on n'est généralement pas conscient de la langue dans laquelle telle ou telle remarque est formulée. C'est qu'on associe directement les désignations aux concepts sans s'arrêter à leurs traits sémiques. Puisque les images originelles sont en quelque sorte court-circuitées au niveau de la conscience, leurs différences dans les deux langues passent tout aussi inaperçues.

À force d'ignorer ces images, on finit par oublier que la désignation nomme seulement un ou deux traits de l'objet, qui le représentent mais qui ne le décrivent pas de façon exhaustive. Ce n'est que lors du premier contact avec un objet et sa désignation qu'on en prend généralement conscience, ou lors de l'apprentissage d'une langue étrangère.

Dans ce dernier cas, on découvre que les mêmes objets ou concepts sont dénommés par des traits différents de ceux qui ont été retenus pour leur désignation dans sa propre langue (exemple : être juge et partie - Richter in eigener Sache sein). Si l'on se sert peu de cette langue étrangère, en d'autres termes si l'on a rarement l'occasion d'associer à l'objet ou au concept leur désignation dans cette langue, les traits nommés restent évocateurs d'images. Je me rappelle, par exemple, avoir été frappée, en apprenant le français, par l'expression les pieds de la table. Non seulement la rencontre avec ces mots m'a fait prendre conscience du fait que Tischbein, en allemand, se réfère bien à un membre du corps - la jambe - mais il m'a aussi fallu un certain temps avant de pouvoir associer les pieds à une table autre que très basse, reposant en quelque sorte seulement sur l'extrémité inférieure du Tischbein. Ce n'est que par l'association fréquente du nom à l'objet que les mots les pieds de la table ont fini par ne plus évoquer, pour moi, que l'objet.

Dans le discours général, les désignations, bien qu'elles ne constituent que des synecdoques, pour reprendre le terme utilisé par M. Lederer ${ }^{4}$,évoquent donc directement l'objet ou le concept tout entier, ce qui permet précisément à l'interprète d'y associer instantanément les désignations appropriées dans la langue cible quoique celles-ci, très souvent, nomment un trait saillant différent.

En entendant une métaphore, en revanche, l'auditeur ne saisit pas seulement l'idée. Il voit aussi l'image suggérée. Il en est de même pour l'interprète. Quand l'image n'est pas directement transposable, il ne parvient pas, dans le temps dont il dispose, à s'en dégager suffisamment pour en faire surgir une autre plus appropriée à la langue cible. Il ne lui reste donc qu'à supprimer la métaphore ou à n'en transmettre que le sens. De ce fait, sa traduction sera incomplète ou se situera à un niveau de langue différent de celui de $1^{2}$ original.

C'est donc la double caractéristique de la métaphore - à savoir sa fonction stylistique qui la tient fermement ancrée dans la langue source, et son pouvoir évocateur d'images trop rémanentes pour être refoulées instantanément au profit du seul message - qui fait obstacle au déroulement du processus de l'interprétation simultanée. Et comme les métaphores sont fréquentes dans les discours littéraires, elles me paraissent responsables, du moins partiellement, de difficultés soulevées, en simultanée, par le registre littéraire. 
Le second obstacle auquel se heurte l'interprétation simultanée des discours littéraires est l'affaiblissement temporaire du sens de la langue que l'on observe chez l'interprète en cabine.

\section{LE SENS DE LA LANGUE}

Nul ne conteste sans doute que, pour réussir un quelconque effet de style, il faut avoir au minimum le sens de cette langue. Or, le sens de la langue maternelle de l'interprète est nettement amoindri en simultanée. Tout interprète le sait pour l'avoir remarqué maintes fois en réunion, mais, à moins de s'enregistrer, il ne l'aura, la plupart du temps, observé que chez son collègue de cabine, et pour cause. En effet, cet affaiblissement du sens de la langue est dû, me semble-t-il, à deux facteurs concomitants : l'interférence entre les langues et le manque de feed-back.

a) Les interférences entre les langues causes :

L'apparition d'interférences linguistiques en traduction peut être due à plusieurs

\section{Compréhension insuffisante du message}

Si, faute de comprendre le sens d'un message, on cherche à traduire le signifié des mots d'une langue dans une autre, les interférences sont inéluctables puisque les langues ne découpent pas la réalité d'une façon identique ni ne désignent les contenus cognitifs par les mêmes traits sémantiques.

- Connaissances insuffisantes du jargon technique dans la langue cible

Lorsque le sujet traité en réunion est technique, par exemple, l'interprète peut ne pas connaître tel ou tel mot du jargon technique qu'il devrait utiliser dans la langue cible. Sur le moment, il ne lui reste donc qu'à traduire le signifié du terme technique. L'interférence linguistique qui en résulte n'est alors pas due à l'incompréhension du sens de l'énoncé. L'interprète a fort bien pu saisir le sens du message, mais il ne maîtrise pas suffisamment le langage spécifique du sujet traité pour réexprimer ce sens dans les termes de métier de la langue cible.

La troisième cause des interférences linguistiques, qui, à ma connaissance, n'a guère été élucidée, est la confusion qui se produit parfois chez des locuteurs bilingues 5 entre les traits retenus par leurs deux langues pour nommer les objets et les concepts.

C'est cette troisième origine des interférences linguistiques qui me paraît expliquer, du moins partiellement, le fait qu'en travaillant en simultanée, l'interprète perd, dans une certaine mesure, le sens de sa langue maternelle. Nous allons donc l'examiner de plus près.

\section{Interférences par suite de permutations des traits pertinents}

Plusieurs observations faites en réunion; dans le cadre de l'enseignement de l'interprétation ou simplement dans ma vie de bilingue, m'ont amenée à m'interroger sur le processus mental qui conduit à des interférences linguistiques en l'absence de difficultés terminologiques ou d'erreur méthodologique en traduction.

En effet, certains interprètes, en travaillant à partir de leur langue passive ${ }^{6}, s^{\prime} e^{-}-$ priment d'une façon beaucoup plus conforme au génie de la langue cible que lorsque la langue source est pour eux une langue active.

Le cas d'un étudiant de l'ÉSIT ${ }^{7}$ illustre ce phénomène. À l'examen, la qualité d'expression de ses interprétations à partir d'une langue active dont il avait une maîtrise remarquable était si faible que les membres du jury se demandaient si le candidat avait 
vraiment une langue maternelle. Ils n'en doutèrent plus lorsqu'ils l'entendirent travailler à partir de sa langue passive.

Même si ces constatations ne permettent pas de conclure avec certitude que c'est parce que la langue source est active qu'elle perturbe davantage le processus de l'expression que ne le fait une langue passive, elles sont pour le moins troublantes.

L'exemple suivant tiré de mon expérience personnelle montre de façon plus précise que le caractère actif de la connaissance d'une deuxième langue joue un rôle important dans l'apparition des interférences dans la langue maternelle.

J'interprétais une réunion où la discussion portait sur l'opportunité de publier un rapport. Un des participants s'exprimait - je le cite de mémoire - à peu près dans ces termes: Si nous publions le rapport maintenant, nous ne ferons que renforcer la position de nos adversaires. Je voulais rendre le sens de cette phrase par : Die Veröffentlichung dieses Berichtes wäre derzeit für unsere Gegner Wasser auf die Mühle. Avant d'articuler la deuxième partie de cette phrase, je m'aperçus que je m'apprêtais à dire Öl auf die Mühle. Devant cette absurdité que, sur le moment, je ne réussis pas à l'éliminer par l'évocation du terme adéquat, à savoir Wasser, il ne me restait aucune autre solution que d'utiliser une expression différente.

On voit que ce n'est pas la tournure employée par l'orateur qui a provoqué l'interférence, puisque huile sur le feu n'a pas été prononcé. Il s'agit plutôt d'un mélange entre deux formules comme il s'en produit fréquemment à l'intérieur d'une même langue, la particularité étant, en l'occurrence, qu'il y a eu mélange entre deux locutions appartenant à deux langues différentes.

On peut se demander comment huile sur le feu a pu me venir à l'esprit alors qu'il aurait été tellement plus logique, compte tenu de la tournure allemande, de penser à apporter de l'eau au moulin. La seule explication, à mon avis, est que cette dernière locution m'était connue, certes, mais contrairement à la première, ne faisait pas partie de celles que j'utilisais spontanément. En d'autres termes, je n'en avais pas une connaissance aussi active.

Une autre observation m'a frappée récemment. Une amie me téléphone pour me demander l'équivalent allemand de mouton retourné. Je lui réponds aussitôt que je peux lui en indiquer deux : Lammfell et un second qui a quelque chose à voir avec de la laine. Alors que je suis sûre de connaître ce mot, je le cherche en vain dans ma mémoire. $\mathrm{Ce}$ n'est qu'après avoir changé de sujet de conversation que je le trouve ; seulement, ce n'est pas un mot allemand mais le terme français peau lainée.

Je crois que ce dernier exemple montre, en quelque sorte au ralenti, comment se produisent les interférences entre des langues qui sont toutes deux utilisées de façon active. Voici mon hypothèse :

On possède une langue active lorsqu'on peut, à tout moment, évoquer, dans cette langue, les tournures et termes convenant aux idées qu'on désire exprimer. La récupération d'un signe dans la mémoire implique la capacité d'évoquer et le signifiant et le signifié de ce signe.

Comme nous l'avons vu à propos des métaphores, le signifié ne donne pas une description exhaustive de l'objet ou du concept qu'il désigne, mais nomme seulement un de ses traits. Pour pouvoir utiliser le signe, il faut que ce trait ait, dans la mémoire du locuteur, un statut privilégié parmi les autres attributs de l'objet. Ainsi l'objet appartement est nommé en français par son attribut de séparation. Ce n'est pas pour autant le seul trait que celui qui connaît l'objet appartement associe à ce dernier. Il sait qu'il sert d'habitation, qu'il se compose de plus d'une pièce, etc. Il se peut même que l'individu, selon son expérience, soit davantage conscient des autres sèmes que du trait dénominateur. Mais pour l'évocation du signe appartement, les autres attributs n'ont pas la même per- 
tinence que celui de la séparation. Ils doivent donc s'effacer devant ce dernier afin de permettre le rappel du signe.

En partant de l'hypothèse établie par J.-F. Le $\mathrm{Ny}^{8}$ sur l'organisation sémantique des lexèmes, on pourrait dire en schématisant que dans la mémoire de celui qui possède une langue active, les traits par lesquels cette langue désigne les objets et les concepts ont un relief plus fort que les autres attributs de ces objets ou concepts.

Il est concevable que ce relief soit engendré par la sollicitation fréquente du trait pertinent qui intervient à chaque emploi du signe. Ceci expliquerait que pour acquérir une langue active, il faut s'en servir presque constamment.

Si la possession d'une langue active signifie que les traits pertinents pour la désignation des objets et des concepts dans cette langue ont pris un relief suffisant pour l'évocation des signes, la possession de deux langues actives implique l'existence d'un double relief verbal. Pour reprendre l'exemple de l'appartement : dans la mémoire du sujet bilingue français-allemand, ce n'est pas seulement l'attribut de séparation qui se profile en saillie, mais aussi l'attribut d'habitation (Wohnung).

Or, il ne me semble pas que les reliefs verbaux soient étiquetés comme appartenant à la langue $\mathrm{A}$ ou à la langue $\mathrm{B}$. Au moment de l'acte de la parole, ils ne se signalent donc pas clairement au locuteur comme le trait pertinent du système linguistique $\mathrm{A}$ ou $\mathrm{du}$ système $B$.

C'est cet anonymat des reliefs verbaux dans la mémoire du sujet parlant, qui, à mon avis, est responsable des interférences entre les langues. En s'exprimant dans une langue $\mathrm{A}$, le locuteur peut, par moments, se tromper de relief verbal, c'est-à-dire solliciter le trait pertinent de la langue $B$.

Au niveau des signifiants, en revanche, les langues A et $\mathbf{B}$ sont bien séparées l'une de l'autre dans la conscience du locuteur. En effet, si l'on utilise un mot français dans une phrase allemande, on peut le faire délibérément ou par négligence, mais on ne peut pas ne pas s'en rendre compte. Par conséquent, lorsqu'un locuteur bilingue employant la langue A se trompe de relief verbal, il s'aperçoit de son erreur au moment où il s'apprête à prononcer l'expression empruntée à la langue $\mathrm{B}$. Ayant identifié le signifiant de la langue $\mathrm{B}$ comme étant étranger à la langue $\mathrm{A}$, il a tendance à le remplacer par son homologue en langue $A$. En fait, il fabrique un nouveau signe dont le signifiant appartient à la langue $\mathrm{A}$ et la signification à la langue $\mathrm{B}$.

Voici un exemple relevé dans une interprétation du français en allemand :

Enfin, un autobus expérimental à propulsion électrique a pu être réalisé.

\author{
... und gleichzeitig ist ein \\ elektrisch angetriebenes \\ Versuchsfahrzeug augenblicklich \\ in Untersuchung.
}

En disant ist in Untersuchung l'interprète, de toute évidence se trompe de relief et aboutit à l'expression française est à l'étude qu'il convertit in extremis en ist in Untersuchung.

Or, l'orateur n'a pas utilisé la tournure est à l'étude. L'interférence n'est donc pas due à une transposition littérale de la forme originale du message, mais à une permutation des reliefs verbaux au cours de la phase de réexpression. Il s'agit, on le voit, non pas d'un problème de traduction mais d'un accident au niveau de l'expression.

Vu sous cet angle, on comprend aisément pourquoi, en simultanée, la langue cible est davantage malmenée par une langue source active que par une langue source passive. En effet, la perception de la langue source active stimule, dans la mémoire de l'interprète, les traits pertinents correspondant à cette langue, ce qui augmente pour lui le 
risque de se tromper de relief verbal dans la phase de réexpression. Inversement, dans le cas où la langue source est pour lui une langue passive, la perception de celle-ci ne produit pas le même effet puisque la plupart des attributs dénominateurs de cette langue n'ont pas encore acquis un relief suffisant dans sa mémoire.

$\mathrm{Si}$, comme je le pense, les interférences entre deux langues sont dues à une confusion entre deux reliefs verbaux dont le sujet bilingue dispose sans être pleinement conscient de leur appartenance linguistique, on doit s'étonner, au fond, de ne pas les voir se produire beaucoup plus souvent, car, à chaque acte de parole, il ne devrait y avoir que $50 \%$ de chances pour le locuteur de solliciter effectivement les traits pertinents de langue qu'il est en train de parler.

La réalité est naturellement différente, parce que pour l'évocation d'un signe, celle de son signifiant est pour le moins tout aussi essentielle que celle de son signifié. L'efficacité de l'indice phonétique pour la récupération de mots sur le bout de la langue est là pour montrer la part importante qui revient à l'évocation du signifiant dans le rappel d'un signe.

Or, lorsqu'on parle, un signifiant appelle l'autre, soit en vertu de la syntaxe, soit parce que l'on se contente de réciter des expressions toutes faites. Le cas le plus extrême est sans doute celui du numéro de téléphone connu par cour. Les signifiants qui le composent sont comme soudés les uns aux autres, si bien qu'il suffit d'en articuler les premiers pour pouvoir débiter automatiquement tous les autres. En construisant ses phrases, le locuteur est aidé par cet effet d'entraînement qu'un signifiant produit sur le suivant.

C'est ce même effet d'entraînement qui le met à l'abri de bon nombre d'interférences linguistiques puisqu'il agit au niveau des signifiants, et à ce niveau-là, nous l'avons $\mathrm{vu}$, les langues n'ont pas tendance à se mélanger. Un signifiant français n'appelle jamais un signifiant allemand mais toujours un autre signifiant français.

Or, cette cohésion entre les signifiants semble se relâcher en interprétation simultanée, comme le montrent les exemples suivants :
... une partie de la clientèle
se reportant soit vers la voiture
Ein Teil der Fahrgäste stieg
particulière soit vers le métro. entweder auf die Metro über oder zum Privatwagen.

Si la cohésion avait pleinement joué sur l'axe syntagmatique, stieg et auf auraient dû entraîner um (au lieu de über), puis oder auf den Privatwagen um (au lieu de zum).

Le même affaiblissement des forces cohésives entre les signifiants s'observe sur l'axe paradigmatique. En voici deux exemples :

... vous savez que sur l'Atlantique Nord, même avant le retrait du paquebot " France", le transport maritime de passagers par paquebot n'existait pratiquement plus.

Ça n'a rien à voir, mais ça vous donne des ordres de grandeur.
Sie wissen, dass praktisch auf der Nordatlantikroute, selbst bevor das Schiff "France" aus dem Verkehr gezogen worden ist, praktisch überhaupt keine Schiffsgäste mehr existierten auf dem Atlantik.

aber immerhin, es gitbt Ihnen eine Grössenvorstellung.

Le signifiant Schiffs-aurait dû entraîner passagiere-ou éventuellement -reisende, et le signifiant grössen-aurait dû appeler -ordnung. Mais comme la cohésion entre les si- 
gnifiants s'est relâchée, ce sont des termes impropres (-gäste et -vorstellung) qui sont évoqués à leur place, et ce en dépit du fait que le mot passagers figure dans l'original et que Grössenordnung est la traduction littérale de ordre de grandeur.

On voit que l'affaiblissement de la cohésion entre les signifiants prive l'interprète de certains automatismes qui, d'ordinaire, facilitent le processus de l'expression. De ce fait, l'évocation des signes devient plus précaire.

Comment s'explique ce relâchement des forces cohésives entre les signifiants ? C'est à mon avis le manque de feed-back qui est la cause principale.

b) Le manque de feed-back

D'après les travaux de nombreux chercheurs', il semble en effet que l'évocation des signifiants exige une certaine participation de l'oreille.

En d'autres termes, pour le locuteur, la perception normale de sa voix et de ses paroles joue un rôle important dans le déroulement correct du processus de l'expression.

Or, l'interprète en simultanée s'entend mal. Certes, il essaie de partager son potentiel d'écoute entre l'original et ses propres paroles, mais compte tenu de l'importance que revêt l'écoute concentrée dans le processus d'interprétation, c'est sur l'orateur que son oreille est branchée en premier lieu. En fait, il n'entend pas vraiment ce qu'il dit, même s'il s'astreint à prêter une certaine attention à ses propres paroles.

J'ai acquis cette conviction non seulement en écoutant travailler des collègues et des étudiants, mais surtout après m'être enregistrée périodiquement en cabine pendant plusieurs années. Le nombre de lapsus linguae non corrigés, dont je ne m'étais donc pas aperçue en interprétant, m'a persuadée qu'en traduction simultanée, le feed-back est sinon inexistant, du moins extrêmement faible.

À force de m'enregistrer en cabine et de corriger a posteriori les anomalies de la forme que je n'avais pas remarquées en travaillant, j'ai progressivement pu en diminuer la fréquence. Il semble donc qu'on puisse réduire délibérément l'incidence du contrôle auditif.

Reste à savoir comment s'opère cette désensibilisation partielle et graduelle du locuteur au manque de feed-back. Les enregistrements réalisés en cabine ne servent sans doute que de révélateurs des imperfections. Du moment qu'il a pris conscience des effets que l'insuffisance de feed-back produit sur sa parole, l'interprète développe peut-être davantage ce que Kainz ${ }^{10}$ appelle la Diktion, c'est-à-dire la formulation intérieure.

Mais le processus de désensibilisation est probablement bien plus complexe. Il mériterait, à mon avis, d'être examiné à fond, car la connaissance des mécanismes en cause permettrait sans doute de mettre au point des armes plus spécifiques et plus efficaces que ne l'est l'emploi du magnétophone pour combattre l'affaiblissement du sens de la langue maternelle en interprétation simultanée.

En conclusion, si l'interprète parvenait à mieux se prémunir contre l'incidence d'un contrôle auditif insuffisant et contre les interférences linguistiques, c'est assurément la traduction simultanée des discours littéraires qui en serait la première bénéficiaire. Certes, tous les problèmes ne seraient pas résolus pour autant. Celui des métaphores et de la quasi-incompatibilité de l'improvisation avec le registre littéraire resterait entier. Mais ce n'est pas parce que la version interprétée en simultanée n'égalera probablement jamais en élégance stylistique le texte original - surtout s'il n'y a pas eu lecture préalable - qu'il faut baisser les bras. Mieux vaut y voir un défi et le relever au nom de la fidélité à laquelle l'interprète reste tenu envers et contre tout. 
Notes

1. Selon la classification des niveaux de langue adoptée par F. Vanoye, dans Expression - Communication, p. 31.

2. Cette remarque ne s'applique bien entendu qu'au cas où l'orateur parle sa langue maternelle et est, de ce fait, effectivement maître du choix du registre.

3. Extrait d'un discours lu en séance.

4. Dans "Implicite et explicite", in Interpréter pour traduire, p. 38.

5. J'entends par bilingues des personnes sachant s'exprimer spontanément et avec aisance dans deux langues différentes, qui, de ce fait, sont pour eux des langues actives.

6. Langue qu'on comprend mais dont la majorité des termes et des tournures ne vient pas assez promptement à l'esprit pour permettre une expression spontanée des idées sous une forme qui correspond à l'usage.

7. École supérieure d'interprètes et de traducteurs de l'Université de Paris (III).

8. "Sémantique et psychologie", Langages, 40, p. 26.

9. Entre autres Denes et Pinson, Cherry, Schubenz, Stolz, Tannenbaum.

10. Psychologie der Sprache, t. III, pp. 142 et 149.

\section{BIBLIOGRAPHIE}

CHERRY, C. (1971) : On Human Communication, 2nd ed., Cambridge, Mass., London, The M.I.T. Press, $337 \mathrm{p}$.

DÉJEAN LE FÉAL, K. (1978) : Lectures et improvisations. Incidences de la forme de l'énonciation sur la traduction simultanée (français-allemand), thèse de doctorat de $3^{e}$ cycle, Université de la Sorbonne Nouvelle (Paris III), 195+A78 p.

DÉJEAN LE FÉAL, K. (1976) : "Le perfectionnement linguistique ", Études de linguistique appliquée, 24, Paris, Didier, pp. 42-51.

DENES, P.B. et E.N. PINSON (1973) : The Speech Chain, New York, Anchor Press, 217 p.

KAINZ, F. (1965) : Psychologie der Sprache, t. I-V, Stuttgart, Encke, 2626 p.

LEDERER, M. (1981) : la Traduction simultanée : expérience et théorie, Paris, Minard, $454 \mathrm{p}$.

LE NY, J.-F. (1975) : " Problèmes de sémantique psychologique ", Langages, 40, Paris, Didier-Larousse, pp. 3-29.

LIEURY, A. (1975) : la Mémoire, Bruxelles, Dessart et Mardaga, 265 p.

MARFELD, A.F. (1973) : Kybernetik des Gehirns, Reinbek bei Hamburg, Rowohlt Taschenbuch, 380 p.

NATAF, R. (1974) : "L'écrit littéraire ", le Français dans le monde, 109, Paris, Hachette-Larousse, pp. 45-48.

PORZIG, W. (1967) : Das Wunder der Sprache, Bern, München, Francke, 424 p.

SAUVAGEOT, A. (1962) : Français écrit, français parlé, Paris, Larousse, 233 p.

SCHUBENZ, S. (1965) : Beitrag zu einer Analyse der selbsttätigen Steuerungsvorgänge im Sprechverhalten, untersucht mit der Technik der künstlichen Verzögerung der auditiven Rückkopplung, InauguralDissertation, Berlin, Dissertations-Druckstelle, $275 \mathrm{p}$.

SELESKOVITCH, D. (1968) : l'Interprète dans les conférences internationales - problèmes de langage et de communication, Paris, Lettres modernes, $261 \mathrm{p}$.

SELESKOVITCH, D. (1975) : Langage, langues et mémoire, Paris, Lettres modernes, 272 p.

SELESKOVITCH, D. et M. LEDERER (1984) : Interpréter pour traduire, Paris, Didier Érudition, $311 \mathrm{p}$. STOLZ, W.S. et P.H. TANNENBAUM (1963) : "Effects of Feedback and Oral Encoding Behaviour ", Language and Speech, 6, pp. 222-226.

TABOURET-KELLER, A. (1974) : "L'écrit. Quelques considérations psychologiques , le Français dans le monde, 109, Paris, Hachette-Larousse, pp. 17-20.

THIERY, C. (1975) : le Bilinguisme chez les interprètes de conférence professionnels, thèse de doctorat de $3 \mathrm{e}$ cycle, Université de la Sorbonne Nouvelle (Paris III), $166 \mathrm{p}$.

VANOYE, F. (1973) : Expression - Communication, Paris, Armand Colin, 242 p. 